\title{
Electronic Bases of Molecular Vibrations. I. General Theory for Diatomic Molecules* $\dagger$
}

\author{
Paul Phichroson \\ Harrison M. Randall Laboratory of Physics, University of Michigan, Ann Arbor, Michigan
}

(Received 19 July 1963)

\begin{abstract}
The force constant of a diatomic molecule in a given electronic state is the second derivative with respect to the internuclear distance $R$ of the molecular electronic energy, evaluated at the equilibrium distance $R_{\text {. }}$. In principle it is determined by the electronic-charge distribution $\psi^{*} \psi$, where $\psi$ is the appropriate solution of the electronic-wave equation. Expressions for the force constant in terms of the electronic-charge distribution are derived which differ depending upon (1) what coordinate representation is used to describe the wavefunction, (2) whether the virial theorem or the Hellmann-Feynman theorem is employed to define the first energy derivative. Correspondingly, if the wavefunction is expressed in confocal elliptic coordinates, two different expressions for the force constant are obtained. These expressions give identical results for exact wavefunctions or for approximate wavefunctions which are constructed to satisfy the virial theorem for all $R$. If this condition is not satisfied, the two expressions will give, in general, different results. It is shown that if the Hellmann-Feynman theorem is used to define the first energy derivative, then the force constant assumes a particularly simple form which is suggestive of the force constant of a classical harmonic oscillator. The utility of the force constant expressions as probes to explore molecular electroniccharge distributions is discussed and related problems are suggested.
\end{abstract}

\section{INTRODUCTION}

$\mathbf{I}^{\mathrm{T}}$ $\mathrm{T}$ is well known that once the electronic wavefunction for a given molecular electronic state is specified, any property of the molecule in this state can in principle be numerically determined by computing the expectation value of the appropriate operator over the electronic-charge distribution. Within recent years many crucial electric and magnetic molecular properties, such as dipole moments, electronic polarizabilities, spin parameters, and quadrupole coupling constants, have been computed to varying degrees of reliability, dependent upon the accuracy of the molecular wavefunctions employed. ${ }^{1}$ These computations have been significant not only for the values of the quantities they yield, but also for the insights they give concerning the details of the electronic-charge distribution characterizing a given molecular state.

Little attention has been paid, however, to similar $a b$ initio calculations of molecular force constants. Indeed, the force-constant operator involved has never been stated in a form which would facilitate calculation, nor have those features of the electronic charge density to which the force constant is most sensitive been systematically investigated. In 1950, Platt ${ }^{2}$ derived an approximate theoretical expression for the force constant of a diatomic molecule and applied it with some success to diatomic hydrides. Platt's result was criticized by Clinton, ${ }^{3}$ who noted that Platt's derivation

* A report of this work was presented at the Symposium on Molecular Structure and Spectroscopy, June 1963, Columbus, Ohio.

$\uparrow$ Supported in part by the Office of Naval Research, Department of the Navy.

\$Present address: Department of Physics, University of Colorado, Boulder, Colorado.

1 For example, see B. J. Ransil, Rev. Mod. Phys. 32, 239, 245 (1960).

${ }^{2}$ J. R. Platt, J. Chem. Phys. 18, 932 (1950).

3 W. L. Clinton, J. Chem. Phys. 33, 1603 (1960). ignored the explicit variation of the electronic-charge density with internuclear distance. On the other hand, by differentiation of the virial theorem ${ }^{4}$ with respect to the internuclear distance $R$, Clinton concluded that the explicit variation of the electronic-charge density with internuclear distance is a necessary precondition for the molecule to have a nonvanishing force constant at all. Platt's impressive numerical results were interpreted as due to approximate cancellation of higher correction density terms. The fact that the force constant involves the derivative of the electronic-charge density with respect to $R$ was shown earlier by Brown ${ }^{5}$ through differentiation of the Hellmann-Feynman theorem. ${ }^{6}$ Brown expanded the derivative of the molecular wavefunction in terms of a complete orthonormal set and proceeded to express the force constant in terms of infinite perturbation sums. More recently, Salem $^{\text {7a }}$ has systematically investigated infinite sums such as arise in Brown's formulation and has derived interesting sum rules. Finally, in a later paper, ${ }^{7 \mathrm{~b}} \mathrm{Salem}$ employed these rules to arrive at various alternative expressions for the force constant.

The primary purpose of the present paper is to investigate further the mathematical forms which the force constant can assume. The form depends upon (1) whether the virial theorem or the Hellmann-Feynman theorem is used to define the first energy derivative, and (2) what coordinate representation is employed to describe the wavefunction. While the first point was emphasized by Salem ${ }^{7 b}$ the second has not been considered. It will be shown here, however, that the choice of coordinate representation is an equally im-

\footnotetext{
${ }^{4} \mathrm{H}$. Eyring, J. Walter, and G. Kimball, Quantum Chemistry (John Wiley \& Sons, Inc., New York, 1944), p. 355.

${ }^{5}$ W. Byers Brown, Proc. Cambridge Phil. Soc. 54, 257 (1958).

'R. P. Feynman, Phys. Rev. 56, 340 (1939).

${ }^{7}$ L. Salem, (a) Phys. Rev. 125, 1788 (1962); (b) J. Chem. Phys. 38, 1227 (1963).
} 
portant factor: changing the coordinate representation will change the form of the force constant expression and its possible interpretation. Furthermore, the procedure to be followed will attempt to emphasize logical connections between the virial theorem and HellmannFeynman approaches. In particular, it is demonstrated that if the wavefunction is exact in the sense that the virial theorem is satisfied for all internuclear distances $R$, and if the wavefunction is expressed in confocal elliptic coordinates, then one expression for the force constant expression admits of a classical mechanical interpretation. This hopefully will put one in a better position to explicitly analyze the role which the electronic-charge distribution plays in the vibrations of atomic nuclei. One question which may be asked is, for example, to what features of the electronic-charge density are molecular vibrations most sensitive? Also, how important are the effects of electron correlation, and the related question of how reliable are HartreeFock (best uncorrelated) wavefunctions ${ }^{8}$ to provide the charge-density ambient in which the nuclei move?

It is to these and related questions that this paper purports to be an introduction. In general, for a diatomic molecule, the force constant is the second derivative of the total molecular electronic energy with respect to $R$, evaluated at the equilibrium distance $R_{e}$. The nature of the differentiation process is investigated in the next section. Consideration of the first energy derivative yields the mathematical conditions under which the Hellmann-Feynman theorem ${ }^{6}$ is satisfied. These conditions, while always met for exact eigenfunctions of the molecular electronic Hamiltonian, ${ }^{9}$ are in practice not usually satisfied for approximate wavefunctions. This situation has been recognized most notably by Hurley ${ }^{10}$ and is re-emphasized here. The Hellmann-Feynman theorem having been obtained, then the differentiation process is applied again to arrive at the second energy derivative, and hence the force constant. At this point it appears that, subject to the conditions previously mentioned, not only does the first energy derivative have a simple classical (electrostatic) interpretation, ${ }^{6,11}$ but also that the diatomic molecular force constant has a direct classical (mechanical) interpretation. Furthermore, a second expression for the force constant is derived which involves essentially differentiation of the virial theorem and comparison made with the first expression. The discussion concludes with consideration of the problem of

${ }^{8}$ C. C. J. Roothaan, Rev. Mod. Phys. 23, 69 (1951).

$\rightarrow$ The subsequent discussion assumes that only the electrostatic portions of the Hamiltonian be considered, with spin and orbital coupling terms neglected. The latter would introduce deviations from the Hellmann-Feynman theorem. See, for example, J. O. Hirschfelder, C. F. Curtiss, and R. B. Bird, Molecular Theory of Gases and Liquids, (John Wiley \& Sons, Inc., New York, 1954), p. 933 .

${ }^{10}$ A. C. Hurley, Proc. Roy. Soc. (London) A226, 170, 179, 193 (1954).

${ }^{11} \mathrm{~J}$. R. Platt, Encyclopedia of Physics (Springer-Verlag, Berlin, 1961), Vol. XXXVII/2, p. 179. $a b$ initio calculation of diatomic force constants when approximate wavefunctions which do not satisfy the Hellmann-Feynman theorem are employed.

\section{DIFFERENTIATION PROCESS AND THE HELLMANN-FEYNMAN THEOREM}

In general, the electronic wavefunction for a diatomic molecule is expressed in terms of orthogonal confocal elliptic coordinates $\left(\xi_{i}, \eta_{i}, \phi_{i}\right)$, where $\phi_{i}$ is the azimuthal angle of the $i$ th electron about the internuclear axis defined by $R$, and $\left(\xi_{i}, \eta_{i}\right)$ are given by

$$
\begin{array}{ll}
r_{a i}=\frac{1}{2}\left(\xi_{i}+\eta_{i}\right) R, & \cos \theta_{a i}=\left(1+\xi_{i} \eta_{i}\right) /\left(\xi_{i}+\eta_{i}\right), \\
r_{b i}=\frac{1}{2}\left(\xi_{i}-\eta_{i}\right) R, & \cos \theta_{b i}=\left(1-\xi_{i} \eta_{i}\right) /\left(\xi_{i}-\eta_{i}\right) .
\end{array}
$$

$r_{a i}$ and $r_{b i}$ are the distances of the $i$ th electron from nuclei $a$ and $b$, respectively; $\theta_{a i}$ and $\theta_{b i}$ are the angles which $r_{a i}$ and $r_{b i}$ make with the internuclear axis $R$. The volume element for the $i$ th coordinates is

$$
d \tau_{i}=\left(R^{3} / 8\right)\left(\xi_{i}^{2}-\eta_{i}^{2}\right) d \xi_{i} d \eta_{i} d \phi_{i}
$$

and the ranges of integration are $1 \leq \xi_{i} \leq \infty,-1 \leq \eta_{i} \leq$ $+1,0 \leq \phi_{i} \leq 2 \pi$. The position vectors $r_{a i}, r_{b i}$ (and hence $\theta_{a i}$ and $\theta_{b i}$ ) are themselves functions of $R$, the functional relations being

$$
\begin{aligned}
z_{a i} & =\left[m_{b} /\left(m_{a}+m_{b}\right)\right] R-z_{i}, \\
z_{b i} & =\left[m_{a i} /\left(m_{a}+m_{b}\right)\right] R+z_{i}, \\
z_{a i}+z_{b i} & =R, \\
r_{a i} & =\left[x_{a i}{ }^{2}+y_{a i}{ }^{2}+z_{a i}{ }^{2}\right]^{1}, \\
\cos \theta_{a i} & =z_{a i} / r_{a i}, \\
r_{b i} & =\left[x_{b i}{ }^{2}+y_{b i}{ }^{2}+z_{b i}{ }^{2}\right]^{3}, \\
\cos \theta_{b i} & =z_{b i} / r_{b i}, \\
x_{a i}{ }^{2}+y_{a i}{ }^{2} & =x_{b i}{ }^{2}+y_{b i}{ }^{2}=x_{i}{ }^{2}+y_{i}{ }^{2},
\end{aligned}
$$

where $\left(x_{i}, y_{i}, z_{i}\right)$ are the coordinates of the $i$ th electron referred to the center of mass of the nuclei of masses $m_{a}$ and $m_{b}$. Thus $\xi_{i}$ and $\eta_{i}$ are explicitly proportional to $R^{-1}$ and implicitly dependent upon $R$ through $r_{a i}$ and $r_{b i}$. Consider an $N$-electron function $G(\xi, \eta, \phi, \alpha)$ where $\xi$ refers collectively to $\xi_{1}, \xi_{2}, \cdots \xi_{N}$ and similarly for $\eta$ and $\phi ; \alpha$ refers collectively to any set of parameters $\alpha_{1}, \alpha_{2}, \cdots$ which are also (implicit) functions of $R$. Then the derivative of $G$ with respect to $R$ is

$$
\frac{d G}{d R}=\frac{\partial G}{\partial R}+\sum_{i=1}^{N}\left[\frac{\partial G}{\partial \xi_{i}} \frac{\partial \xi_{i}}{\partial R}+\frac{\partial G}{\partial \eta_{i}} \frac{\partial \eta_{i}}{\partial R}\right]=\left[\frac{\partial}{\partial R}+\frac{Q(\xi, \eta)}{R}\right] G
$$

where

$$
\frac{\partial G}{\partial R}=\sum_{i} \frac{\partial G}{\partial \alpha_{i}} \frac{\partial \alpha_{i}}{\partial R}
$$

$Q(\xi, \eta)$ is the sum of single-particle differential opera- 
tors $L_{i}\left(\xi_{i}, \eta_{i}\right)$ and $M_{i}\left(\xi_{i}, \eta_{i}\right)$ :

$$
\begin{aligned}
Q(\xi, \eta) & =\sum_{i=1}^{N} Q_{i}\left(\xi_{i}, \eta_{i}\right) \\
& =\sum_{i=1}^{N}\left[L_{i}\left(\xi_{i}, \eta_{i}\right)+\Delta M\left(\xi_{i}, \eta_{i}\right)\right] \\
& =L(\xi, \eta)+\Delta M(\xi, \eta),
\end{aligned}
$$

which from (1) and (2) are given by

$$
\begin{aligned}
L_{i}\left(\xi_{i}, \eta_{i}\right) & =\left(\xi_{i}{ }^{2}-\eta_{i}\right)^{2}-1\left[\xi_{i}\left(1-\xi_{i}{ }^{2}\right) \frac{\partial}{\partial \xi_{i}}+\eta_{i}\left(\eta_{i}{ }^{2}-1\right) \frac{\partial}{\partial \eta_{i}}\right], \\
M_{i}\left(\xi_{i}, \eta_{i}\right) & \left.=\left(\xi_{i}{ }^{2}-\eta_{i}\right)^{2}\right)^{-1}\left[\eta_{i}\left(\xi_{i}{ }^{2}-1\right) \frac{\partial}{\partial \xi_{i}}+\xi_{i}\left(1-\eta_{i}{ }^{2}\right) \frac{\partial}{\partial \eta_{i}}\right],
\end{aligned}
$$

and

$$
\Delta=\left(m_{b}-m_{a}\right) /\left(m_{b}+m_{a}\right) .
$$

In order to explore the integral properties of these operators for subsequent use, consider any two functions $\Phi$ and $\chi$ which are finite at the boundary. Integration by parts gives

$$
\begin{aligned}
\int \Phi L \chi d \tau & =\sum_{i=1}^{N} \int \Phi L_{i} \chi d \tau \\
& =\sum_{i=1}^{N} \int \prod_{j \neq i}^{N} d \tau_{j} \int \Phi L_{i} \chi d \tau_{i} \\
& =3 \sum_{i=1}^{N} \int \prod_{i}^{N} d \tau_{i} \Phi \chi-\sum_{i=1}^{N} \int \prod_{j \neq i}^{N} d \tau_{j} \int \chi L_{i} \Phi d \tau_{i} \\
& =3 N \int \Phi \chi d \tau-\int \chi L \Phi d \tau,
\end{aligned}
$$

with a similar procedure for the operator $M$. The desired integral relations are therefore

$$
\begin{aligned}
& \int \Phi L \chi d \tau+\int \chi L \Phi d \tau=3 N \int \Phi \chi d \tau \\
& \int \Phi M \chi d \tau+\int \chi M \Phi d \tau=0 \\
& \int \Phi Q \chi d \tau+\int \chi Q \Phi d \tau=3 N \int \Phi \chi d \tau .
\end{aligned}
$$

In particular, setting $\chi=\Phi^{*}$, and noting that the operators are real,

$$
\int \Phi^{*} Q \Phi d \tau=\frac{3}{2} N \int \Phi^{*} \Phi d \tau
$$

and setting $\chi=X \Phi^{*}$ where $X$ is any Hermitian operator

$$
\int \Phi^{*} X Q \Phi d \tau+\int \Phi Q X \Phi^{*} d \tau=3 N \int \Phi^{*} X \Phi d \tau
$$

Now, the Hellmann-Feynman theorem ${ }^{6,2-11}$ states that if the wavefunction $\psi(\xi, \eta, \phi ; \alpha)$ is a normalized eigenfunction of the Hamiltonian $\mathfrak{H C}(\xi, \eta, \phi ; R)=T+V$, then the forces on the nuclei are determined by the expectation value of the derivative with respect to the parameter $R$ of the classical electrostatic potential $V$ over the electronic-charge distribution $\psi^{*} \psi$. To put this in mathematical terms, it is convenient to distinguish between the $R$-dependence which occurs through the parameters $\alpha$ in the wavefunction, and the $R$-dependence which occurs in the operators. The convention is adopled that the $R$-dependence in $\alpha$ is denoted by $R^{\prime}$ and the dependence in the operators by $R$. Then, if $E$ is the total electronic energy of the molecule,

$$
E\left[\alpha\left(R^{\prime}\right), R\right]=\int \psi^{*}\left[\alpha\left(R^{\prime}\right)\right] \mathfrak{F C}(R) \psi\left[\alpha\left(R^{\prime}\right)\right] d \tau,
$$

the force is given by

$$
-\bar{F}=\frac{d E}{d R}=\int \psi * \frac{d V}{d R} \psi d \tau
$$

where $d \mathcal{H} / d R=d V / d R$ since $T$, the electronic kineticenergy operator, is independent of $R$ [see Eq. (18)]. The proof, following Feynman, ${ }^{6}$ is to observe that differentiating the right side of $\mathrm{Eq}$. (10) yields three terms, one of which is the right side of (11) while the other two terms give zero contribution,

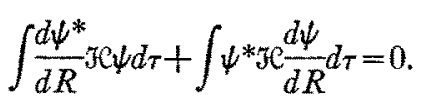

This result follows from the fact that $\psi$ is an eigenfunction of $\mathfrak{H}$ so that the left side of (12) reduces to $E(d / d R) \int \psi^{*} \psi d \tau=E(d / d R)(1)=0$. In general, approximate molecular wavefunctions will not satisfy Eq. (12) although it is possible to construct them such that they will. ${ }^{10}$ Wavefunctions which do fulfill this condition have been called stable wavefunctions by Hall. ${ }^{12}$ It is to be shown here that in the confocal elliptic coordinates representation the condition that the wavefunction be stable is tantamount to the requirement that the variational principle ${ }^{13}$ and the virial theorem be simultaneously satisfied. In other words, for Eq. (12) to hold, the functional representation of $\psi$ in terms of $(\xi, \eta, \phi)$ must be such that the virial theorem holds, while the parameters $(\alpha)$ must assume such values at every internuclear distance such that the variational theorem holds. ${ }^{14}$

To prove this assertion the first step is to express the

${ }^{12}$ G. Hall, Phil. Mag. 6, 249 (1961).

${ }^{13} \mathrm{~L}$. Pauling and E. B. Wilson, Jr., Introduction to Quantum Mechanics (McGraw-Hill Book Company, Inc., New York, 1935), Chap. VII.

14 This statement was essentially made by $\mathrm{J} . \mathrm{O}$. Hirschfelder and C. A. Coulson [J. Chem. Phys. 36, 941 (1962)]. We are going into greater detail here in order to indicate the procedures necessary for subsequent development of the second energy derivative. 
normalized wavefunction as

$$
\psi=\Phi(\xi, \eta, \phi ; \alpha) / S^{\frac{1}{2}}, \quad S=\int \Phi^{*} \Phi d \tau .
$$

Then using Eqs. (3) and (8) and noting the convention noted prior to Eq. (10), the derivative of $\psi$ with respect to $R$ is given by ${ }^{15}$

$$
\frac{d \psi}{d R}=-\frac{1}{2} S^{-1} \frac{d S}{d R} \Phi+\frac{1}{S} \frac{d \Phi}{d R}=\left[\frac{\partial}{\partial R^{\prime}}+\frac{Q(\xi, \eta)}{R}-\frac{3 N}{2 R}\right] \psi
$$

The condition for stability becomes

$$
\begin{gathered}
\int \frac{d \psi^{*}}{d R} \mathfrak{H} \psi d \tau+\int \psi^{*} \mathfrak{H C} \frac{d \psi}{d R} d \tau=\left[\int \frac{\partial \psi^{*}}{\partial R^{\prime}} \mathfrak{C} \psi d \tau+\int \psi^{*} \mathfrak{H} \frac{\partial \psi}{\partial R^{\prime}} d \tau\right] \\
+\frac{1}{R}\left[\int \psi \mathcal{H} Q \psi^{*} d \tau+\int \psi^{*} \mathcal{H} Q \psi d \tau-3 N \int \psi^{*} \mathfrak{H} \psi d \tau\right] .
\end{gathered}
$$

The first bracket is seen to be

$$
\frac{\partial}{\partial R^{\prime}} \int \psi^{*}\left(R^{\prime}\right) \mathfrak{H C}(R) \psi(R) d \tau=\frac{\partial E\left(R, R^{\prime}\right)}{\partial R^{\prime}} .
$$

The last term on the right-hand side of Eq. (15) is eliminated by using Eq. (9) so that the second bracket is equal to

$$
\begin{aligned}
& -\frac{1}{R} \int \psi^{*}[Q \mathcal{H C}-\mathfrak{F} Q] \psi d \tau \\
& =-\frac{1}{R}\left[\int \psi^{*}[Q T-T Q] \psi d \tau+\int \psi^{*}[Q V-V Q] \psi d \tau\right]
\end{aligned}
$$

Thus, the second bracket of Eq. (15) reduces to the commutator of $\mathcal{H}$ and $Q$, which can be seen to vanish if $\psi$ is an eigenfunction of $\mathcal{H}$. To evaluate this commutator, one first notes that in confocal elliptic coordinates the kinetic-energy operator $T$ is explicitly inversely proportional to $R^{2}$ and that the potentialenergy operator $V$ is explicitly inversely proportional to $R{ }^{14} \mathrm{Also}$, since the electronic kinetic energy is independent of $R$, the operator $d / d R$ must commute with $T$ :

$$
(d / d R)(T \psi)=T(d \psi / d R) .
$$

The left and right sides of Eq. (18) are, respectively,

$$
\begin{aligned}
\frac{d}{d R}(T \psi) & =-\frac{2}{R} T \psi+T \frac{\partial \psi}{\partial R}+\frac{(Q T)}{R} \psi \\
T \frac{d \psi}{d R} & =T \frac{\partial \psi}{\partial R}+\frac{(T Q)}{R} \psi
\end{aligned}
$$

so therefore

$$
[Q T-T Q] \psi=2 T \psi
$$

\footnotetext{
${ }^{15}$ In Eq. (14), and in the discussion to follow, it is to be understood that $Q(\xi, \eta)$ operates on $\Phi$ only, while $\partial / \partial R^{\prime}$ operates on $\psi$.
}

The derivative of the potential energy operator is

$$
\frac{d V}{d R}=-\frac{V}{R}+\frac{Q V}{R}
$$

so that

$$
[Q V-V Q] \psi=(Q V) \psi=[R(d V / d R)+V] \psi .
$$

After inserting Eqs. (18) and (19) into (17) and combining the resultant expression with Eq. (16), the condition for stability [Eq. (12)] becomes

$$
\begin{aligned}
\int \frac{d \psi^{*}}{d R} \mathfrak{F} \psi d \tau+\int \psi^{*} \mathcal{H C} \frac{d \psi}{d R} d \tau & \\
= & \frac{\partial E}{\partial R^{\prime}}-\frac{1}{R} \int \psi^{*}[Q \mathcal{F}-\mathcal{F} Q] \psi d \tau
\end{aligned}
$$

where

$$
\frac{\partial E\left[\alpha\left(R^{\prime}\right), R\right]}{\partial R^{\prime}}=\sum_{i} \frac{\partial E}{\partial \alpha_{i}} \frac{\partial \alpha_{i}}{\partial R^{\prime}}
$$

and

$$
\int \psi^{*}[Q \mathcal{H}-\mathcal{H} Q] \psi d \tau=\int \psi^{*}\left[2 T+V+R \frac{d V}{d R}\right] \psi d \tau .
$$

The vanishing of the first term on the right-hand side of Eq. (21) is assured by the variational principle which asserts that the parameters $\alpha_{i}$ must be such that $\partial E / \partial \alpha_{i}=0$ for all $\alpha_{i}$ [Eq. (22)]. Finally, the vanishing of the commutator of $\mathfrak{H C}$ and $Q$ is equivalent to the vanishing of the right side of (23), which is precisely the statement of the quantum-mechanical virial theorem.

To summarize the results, differentiation of the left and right sides of Eq. (10) yields

$\frac{d E}{d R}=\frac{\partial E}{\partial R}=-\frac{1}{R} \int \psi *\left[2 T+V+R \frac{d V}{d R}\right] \psi d \tau+\int \psi * \frac{d V}{d R} \psi d \tau$,

where the term $\partial E / \partial R^{\prime}$ cancels. For exact wavefunctions, that is wavefunctions which are eigenfunctions of the Hamiltonian, the vanishing of the commutator of $\mathfrak{H C}$ and $Q$ is identically the statement of the virial theorem, leaving simply

$$
\frac{d E}{d R}=\frac{\partial E}{\partial R}=\int \psi * \frac{d V}{d R} \psi d \tau \equiv-\bar{F},
$$

which is the Hellmann-Feynman result. If the wavefunctions are approximate in the sense that the virial theorem is not satisfied for all $R, \mathfrak{H C}$ and $Q$ do not commute. In this case the $d V / d R$ terms in Eq. (24) cancel, leaving

$$
\frac{d E}{d R}=\frac{\partial E}{\partial R}=-\frac{1}{R} \int \psi^{*}[2 T+V] \psi d \tau
$$


Of course both Eq. (26) and Eq. (25) are valid for exact eigenfunctions since in the exact case, (26) can be viewed as the result of combined application of the Hellmann-Feynman and virial theorems, viz.,

$\left[\int \psi * \frac{d V}{d R} \psi d \tau=-\bar{F}\right]=\left[-\frac{1}{R} \int \psi *[2 T+V] \psi d \tau=\frac{\partial E}{\partial R}\right]$

The point is that the two brackets are not equal for approximate wavefunctions, for then $\partial E / \partial R$ is not equal to $-\bar{F}^{16}$

\section{FORCE CONSTANT OF A DIATOMIC MOLECULE}

The force constant of a diatomic molecule requires, of course, the determination of the second energy derivative with respect to $R$. For exact wavefunctions, differentiation of the Hellmann-Feynman theorem [Eq. (25)] gives

$$
\begin{aligned}
\frac{d^{2} E}{d R^{2}} & =\frac{\partial^{2} E}{\partial R^{2}}+\frac{\partial^{2} E}{\partial R \partial R^{\prime}} \\
& =\int \frac{d \psi^{*}}{d R} \frac{d V}{d R} \psi d \tau+\int \psi * \frac{d V}{d R} \frac{d \psi}{d R} d \tau+\int \psi^{*} \frac{d^{2} V}{d R^{2}} \psi d \tau .
\end{aligned}
$$

Since

$$
\frac{d^{2} V}{d R^{2}}=-\frac{2}{R} \frac{d V}{d R}+\frac{Q}{R} \frac{d V}{d R}
$$

and again using (14), the right side of (28) becomes

$$
\begin{aligned}
& \frac{\partial}{\partial R^{\prime}} \int \psi^{*} \frac{d V}{d R} \psi d \tau-\frac{2}{R} \int \psi * \frac{d V}{d R} \psi d \tau \\
& +\frac{1}{R}\left[\int \psi^{*} \frac{d V}{d R} Q \psi d \tau+\int \psi Q \frac{d V}{d R} \psi^{*} d \tau-3 N \int \psi^{*} \frac{d V}{d R} \psi d \tau\right] .
\end{aligned}
$$

The bracketed terms vanish by Eq. (9), so that the second energy derivative becomes

$$
\frac{d^{2} E}{d R^{2}}=\frac{\partial^{2} E}{\partial R^{2}}+\frac{\partial^{2} E}{\partial R \partial R^{\prime}}=\frac{2}{R} \bar{F}-\frac{\partial \bar{F}}{\partial R^{\prime}} .
$$

At the equilibrium distance $R=R_{e}$, the force vanishes,

${ }^{16}$ An elementary way to see the differences between Eq. (25) and Eq. (26) for an approximate wavefunction in a simple case is to employ, for the $\mathrm{H}_{2}{ }^{+}$molecule ground state, an approximate representation of the form $\psi \sim \rho^{-\rho \xi}$, where $\rho$ is a parameter. Using Eq. (25), the integrand $\psi^{*}(d V / d R) \psi d \tau$ contains $(\xi+\eta)$ in the denominator, so that the value of the integral contains an exponential integral $E i(-2 \rho)$, as well as a $\ln (2 \rho)$ term [see K. Ruedenberg, C. C. J. Roothaan, and W. Jaunzemis, Technical Report (Laboratory of Molecular Structure and Spectra, University of Chicago, 1952-3), pp. 137-256+ii]. On the other hand, differentiating $E(\rho, R)$ directly with respect to $R$ yields a different, purely algebraic, expression which is identical to the right side of (26).
$\bar{F}=0$, so that the force constant is given by the following equivalent expressions,

$$
\begin{aligned}
k=\left.\frac{d^{2} E}{d R^{2}}\right|_{R_{\boldsymbol{e}}} & =\left.\frac{\partial^{2} E}{\partial R \partial R^{\prime}}\right|_{R_{e}}=-\left.\frac{\partial \bar{F}}{\partial R^{\prime}}\right|_{R_{\boldsymbol{e}}}=-\left.\sum_{i} \frac{\partial \bar{F}}{\partial \alpha_{i}} \frac{\partial \alpha_{i}}{\partial R^{\prime}}\right|_{R_{\boldsymbol{E}}} \\
& =\left[\sum_{i} \frac{\partial \alpha_{i}}{\partial R^{\prime}} \frac{\partial}{\partial \alpha_{i}} \int \psi^{*}\left(\alpha_{i}\right) \frac{d V}{d R^{\prime}} \psi\left(\alpha_{i}\right) d \tau\right]_{R_{e}}
\end{aligned}
$$

This result means that for a diatomic molecule if (1) $\psi$ is expressed in confocal elliptic coordinates [Eq. (1)], and (2) $\psi$ is an eigenfunction such that the HellmannFeynman theorem holds [Eq. (25)], then the force constant may be expressed as the derivative of the Hellmann-Feynman force $(\bar{F})$ with respect to $R$, evaluated at $R=R_{e}$, provided that the derivative acts only on the electronic-charge distribution, i.e., only through the parameters $(\alpha)$. From the third equality in $(30)$ it is seen that the force constant expression is formally similar to that of a one-dimensional classical harmonic oscillator: $F=-k x, k=-d F / d x$. The last equality indicates that determination of the force constant involves first, calculation of the expectation value of the gradient of the potential, secondly the differentiation of the resultant expression with respect to the parameters $(\alpha)$, and finally knowledge of the values at $R=R_{e}$ of the derivatives of these parameters. ${ }^{17}$ The determination of $\left.\left(\partial \alpha_{i} / \partial R\right)\right|_{R e}$ requires, of course, knowledge of the $\alpha_{i}$ 's as a function of $R$. It is here that the satisfying of the variational principle is implied.

As for the first qualification, it is important to note that this form of the force constant is based on a derivation using specifically confocal elliptic-or "twocenter"-coordinates. If, for example, the wavefunction is expressed in terms of electronic coordinates $\left(x_{i}, y_{i}, z_{i}\right)$ referred to the center of mass [see Eq. (2)]- the so-called "one-center" coordinates which are independent of $R$-then the derivative of the wavefunction with respect to $R$ would involve only the derivative with respect to the variational parameters, say $\beta_{i}$. In this case

$$
\psi=\psi(x, y, z ; \beta) ; \frac{d \psi}{d R}=\frac{\partial \psi}{\partial R}=\sum_{i} \frac{\partial \psi}{\partial \beta_{i}} \frac{\partial \beta_{i}}{\partial R} .
$$

The condition for this one-center wavefunction to be stable [Eq. (12)] so that the Hellmann-Feynman theorem holds is only that the variational principle is satisfied. This is a less stringent requirement than the two-center case, which demanded the virial theorem to hold in addition. On the other hand, the force con-

\footnotetext{
17 Of course, one could reverse the procedure and differentiate the charge distribution $\psi^{*}(\alpha) \psi(\alpha)$ first and then evaluate the integral. In practice, however, this is the more laborious procedure, but is useful as a check. This and other computational considerations will be treated in a subsequent communication.
} 
stant in the one-center representation is

$$
k=-\left.\sum_{i} \frac{\partial \bar{F}}{\partial \beta_{i}} \frac{\partial \beta_{i}}{\partial R}\right|_{R_{e}}+\left.\int \psi * \frac{d^{2} V}{d R^{2}} \psi d \tau\right|_{R_{e}} .
$$

The first term of (32) appears formally to be identical with the fourth equality of $(30)$, yet there is no contradiction: the dependence of the one-center parameters $\beta_{i}$ on $R$ is different from the $R$ dependence of the twocenter parameters $\alpha_{i} \cdot{ }^{18}$ Thus the sums, while of similar form, are not the same. The second term in (32) might be viewed as a "quadrupole correction" to the force constant expressed in terms of one-center wavefunctions, with the consequent loss of the simple interpretation characteristic of the two-center case. ${ }^{19}$

It is evident, then, that the degree to which the force constant depends upon explicit variation of the wavefunction with $R$ is contingent on the choice of coordinate representation. Assuming the HellmannFeynman theorem to hold, the dependency is complete in the two-center case but not in the one-center case. One can go even further along these lines and demonstrate that even in the two-center case, if approximate wavefunctions are employed, the dependency of the force constant on variation of the charge density is also not complete. Now Eq. (26), in which the first energy derivative is defined via the virial theorem, must be differentiated. Equation (26) may be written as

$$
\frac{d E}{d R}=-\frac{2 E}{R}+\frac{1}{R} \int \psi^{*} V \psi d \tau
$$

The second energy derivative is

$$
\frac{d^{2} E}{d R^{2}}=-\frac{3}{R} \frac{d E}{d R}+\frac{1}{R} \frac{d}{d R} \int \psi^{*} V \psi d \tau
$$

where $d E / d R$ is given by (33). Using the procedures outlined above, the last term is

$\frac{1}{R} \frac{d}{d R} \int \psi^{*} V \psi d \tau=\frac{1}{R} \frac{\partial}{\partial R^{\prime}} \int \psi^{*} V \psi d \tau-\frac{1}{R^{2}} \int \psi^{*} V \psi d \tau$,

so that

$$
\frac{d^{2} E}{d R^{2}}+\frac{3}{R} \frac{d E}{d R}=\frac{1}{R} \frac{\partial \bar{V}}{\partial R^{\prime}}-\frac{\bar{V}}{R^{2}}
$$

where $\vec{V}$ is the expectation value of the potential energy. At $R=R_{e}, d E / d R=0$, or $2 \bar{T}=-\bar{V}$, so that the

\footnotetext{
${ }_{18}$ C. A. Coulson and A. C. Hurley, J. Chem. Phys. 37, 448 (1962).

${ }_{10}$ The fact that the appearance of the quadrupole operator in the force constant expression is contingent upon the choice of coordinate representation appears to rule out the possibility of assigning an invariant connection between the force constant $k$ and the quadrupole coupling constant $q$, the latter being determined essentially by integrals over the quadrupole operator. Consequently, Salem's equations relating $k$ and $q[\operatorname{Ref} .7(\mathrm{~b})]$ may not be unique.
}

force constant is given by ${ }^{20}$

$$
\begin{aligned}
k & =\left.\frac{d^{2} E}{d R^{2}}\right|_{R_{e}}=\left[\frac{1}{R} \frac{\partial \bar{V}}{\partial R^{\prime}}-\frac{\bar{V}}{R^{2}}\right]_{R_{e}} \\
& =\left[\frac{1}{R} \sum \frac{\partial \alpha_{i}}{\partial R^{\prime}} \frac{\partial}{\partial \alpha_{i}} \int \psi^{*}\left(\alpha_{i}\right) V \psi\left(\alpha_{i}\right) d \tau-\frac{1}{R^{2}} \int \psi^{*} V \psi d \tau\right]_{R_{e}}
\end{aligned}
$$

It should be noted that just as Eq. (26) is as valid for exact wavefunctions as Eq. (25) for the first energy derivative, so Eqs. (36) and (29) are both valid for the second energy derivative.

To summarize, then, the force constant for a diatomic molecule in terms of integrals over the electronic-charge distribution, expressed in confocal elliptic coordinates, is given by

$$
\begin{aligned}
k & =\left.\frac{d^{2} E}{d R^{2}}\right|_{R_{s}}=-\sum_{i}\left[\frac{\partial \bar{F}}{\partial \alpha_{i}} \frac{\partial \alpha_{i}}{\partial R^{\prime}}\right]_{R_{e}} \\
& =\left[\frac{1}{R} \sum_{i} \frac{\partial \bar{V}}{\partial \alpha_{i}} \frac{\partial \alpha_{i}}{\partial R^{\prime}}-\frac{\bar{V}}{R^{2}}\right]_{R_{e}},
\end{aligned}
$$

where

$$
\bar{F}=-\int \psi^{*}(\alpha) \frac{d V}{d R} \psi(\alpha) d \tau ; \quad \bar{V}=\int \psi^{*}(\alpha) V \psi(\alpha) d \tau
$$

Both equalities hold for exact wavefunctions, or, it is to be emphasized, for wavefunctions which are constructed such that both virial theorem and variational principle are satisfied..$^{10}$ These are the conditions for the wavefunction to be stable; and the stability criterion ensures the validity of the Hellmann-Feynman theorem. ${ }^{12}$ If the wavefunctions are approximate

${ }^{20}$ At this point it is important to note the difference between this derivation and that of Clinton (Ref. 3). In place of Eq. (35), the latter author expressed the derivative of $\vec{V}$ as

$\frac{d}{d R} \int \psi^{*} V \psi d \tau=\int V \frac{d}{d R}\left(\psi^{*} \psi\right) d \tau+\int \psi^{*} \frac{d V}{d R} \psi d \tau$

$$
=\int V \frac{d}{d R}\left(\psi^{*} \psi\right) d \tau+\frac{d E}{d R},
$$

where in the last equality the Hellmann-Feynman theorem is assumed. In this case the force constant would be given by

$$
k=\left[\frac{1}{R} \int V \frac{d}{d R}\left(\psi^{*} \psi\right) d \tau\right]_{R^{\prime}}
$$

which would appear to indicate that $k$ depends exclusively on variation of the electronic charge density. However, using the procedures described above,

$$
\int V \frac{d}{d R}\left(\psi^{*} \psi\right) d \tau=\frac{\partial \bar{V}}{\partial R^{\prime}}-\frac{\bar{V}}{R}-\int \psi^{*} \frac{d V}{d R} \psi d \tau
$$

If the last term is identified with $d E / d R$ (by the HellmannFeynman theorem) and this expression substituted into the top expression, then Eq. (37) is obtained. The apparent difference, therefore, resides in the distinction between the $d / d R$ and $\partial / \partial R^{\prime}$ operators. 
in the sense that the virial theorem is not satisfied, then only the second equality holds.

\section{SUMMARY}

The major conclusion to be drawn from the preceding discussion is that the force-constant expression is by no means unique, but on the contrary depends upon the coordinate representation as well as upon whether one takes as the point of departure the HellmannFeynman theorem or the virial theorem, that is, the first or second bracket in Eq. (27). Using the Hellmann-Feynman theorem the force constant is given by the first equality in (38), while differentiation of the virial theorem yields the second equality. To reemphasize the logical relationship between the two expressions, for exact wavefunctions the two statements of the force are both true, since it is immaterial whether Eq. (25) (the Hellmann-Feynman theorem) or Eq. (26) (the virial theorem) be used to define the first derivative. However, if the wavefunction is approximate in the sense that the virial theorem is not satisfied, then one cannot identify the first energy derivative with the Hellmann-Feynman force; it must be identified with Eq. (26). One thus has the odd situation that "if the virial theorem is not satisfied, then the first energy derivative can only be expressed by the virial theorem!" In any event, the force constant is a function of the parameters and parameter derivatives,

$$
k=k\left[\alpha_{i},\left(\partial \alpha_{i} / \partial R\right), R\right]_{R e},
$$

and the determination of their values implies satisfying the variational theorem.

This situation, in which for the exact wavefunction two expressions yield identical results while giving different results for an approximate wavefunction, is a well-known one. For example, a similar circumstance arises in the case of the dipole transition moment. There are three equivalent dipole operators which give identical transition moment values when exact wavefunctions for the initial and final transition states are employed, although the expressions themselves appear quite different. ${ }^{21}$ However, when approximate

${ }^{21}$ H. A. Bethe and E. E. Salpeter, Encyclopedia of Physics (Springer-Verlag, Berlin, 1957), Vol. XXXV, p. 334; S. Chandrasekhar, Astrophys. J. 102, 223 (1945). wavefunctions are used, the values of the oscillator strengths calculated the three ways may differ widely, so that a comparison allows one a sensitive check on the goodness of a pair of approximate molecular wavefunctions. ${ }^{22}$ This comparative method is a rather more critical test of the reliability of an approximate wavefunction than the relatively insensitive energy criterion, since the three operators tend to selectively emphasize different spatial regions of the electronic-charge distribution. The suggestion to be made here is that the force constant expressions may be put to similar use. Namely, using a given approximate wavefunction, the force constant could be computed using both expressions, and the results compared with each other and with experiment. In this way the questions raised in the introduction could be explored.

A second conclusion is that the degree to which the force constant depends upon explicit variation of the wavefunction with $R$ via the parameters is variable. The dependency is complete in the two-center representation assuming the Hellmann-Feynman theorem. However, it is incomplete if the virial theorem only is used in the two-center case, or when the HellmannFeynman theorem is assumed in the one-center case. ${ }^{23}$ Consequently, there arises the general problem of the relative importance of the parameter derivatives $\left[\left(\partial \alpha_{i} / \partial R\right),\left(\partial \beta_{i} / \partial R\right)\right]$ in the expressions for the force constant. Their qualitative and quantitative roles are far from clear and may well vary from one specific molecular case to another, as is often the case for the parameters themselves.

The issues raised here, with application to specific cases, will be the subject matter of future communications.

\section{ACKNOWLEDGMENT}

The author wishes to sincerely thank Professor K. T. Hecht of the Department of Physics, University of Michigan, for his continued interest and suggestions on this work.

\footnotetext{
${ }^{22}$ For further discussion of these points, see S. Ehrenson and P. E. Phillipson, J. Chem. Phys. 34, 1224 (1961).

${ }^{23}$ Indeed, Platt's result (Ref. 2) might be interpreted as an approximation of Eq. (37) in which he in effect neglected the sum term and approximated the $-\bar{V} / R^{2}$ term.
} 